\title{
A QUADRATIC SYSTEM WITH A NONMONOTONIC PERIOD FUNCTION
}

\author{
CARMEN CHICONE AND FREDDY DUMORTIER
}

(Communicated by George R. Sell)

\begin{abstract}
For a certain $c_{*}>1.4$ and $c \in\left(1.4, c_{*}\right)$ the quadratic system $\dot{x}=-y+x y, \dot{y}=x+2 y^{2}-c x^{2}$ has a center at the origin surrounded by a one-parameter family of periodic trajectories. We show the period is not a monotone function of the parameter.
\end{abstract}

1. Introduction. Consider a smooth vector field $X$ on $\mathbf{R}^{2}$ which has a center at the origin surrounded by a family of periodic trajectories and let $\Sigma$ denote a Poincaré section for the flow of $X$ such that $\Sigma$ is transverse to each member of the family of periodic orbits. We choose a regular coordinate $\alpha$ along $\Sigma$ so $\alpha=0$ corresponds to the outer boundary of the family of periodic trajectories (perhaps $L=\infty)$. Then we define the period function $P:(0, L) \rightarrow \mathbf{R}$ to be the function which assigns to each $\alpha \in(0, L)$ the minimum period of the trajectory crossing $\Sigma$ at $\alpha$. During the past several years there has been a substantial amount of work devoted to understanding the behavior of the period function. In particular, several papers study conditions which insure $P$ is a monotone function $[\mathbf{1 - 4 , 6}, \mathbf{8}-14]$. One aspect of this analysis suggests that when $X=F(x, y) \partial / \partial x+G(x, y) \partial / \partial y$ and $F, G$ are quadratic polynomials then if a center exists the corresponding period function is monotone. For example Chow and Sanders show that vector fields which arise from one degree of freedom Hamiltoniar, systems with cubic potentials, i.e., systems of the form $\ddot{x}+g(x)=0$, with $g(x)$ quadratic, necessarily have monotone period functions. Also, several authors $[\mathbf{8}, \mathbf{1 4}]$ have shown all Volterra-Lotka systems of the form

$$
\dot{x}=x(a-b y), \quad \dot{y}=y(c x-d)
$$

have monotone period functions. Thus it seems natural to ask if all quadratic systems behave in this way (cf. [6, p. 11]). The purpose of this note is to provide an example which shows that the behavior of the period function for quadratic systems is more complicated. In particular, our main result is

THEOREM A. The quadratic system $X$ given by

$$
\dot{x}=-y+x y, \quad \dot{y}=x+2 y^{2}-c x^{2} ; \quad 0<c<2,
$$

Received by the editors October 6, 1986.

1980 Mathematics Subject Classification (1985 Revision). Primary 58F22, 34C15, 34C25.

The work of the first author was supported in part by a grant from the Research Council of the Graduate School of the University of Missouri and a grant from the Brazilian National Science Council CNPq.

The work of the second author was supported in part by a grant from the Belgian National Science Foundation NFWO and the Brazilian National Science Council CNPq. 
has a center at the origin surrounded by a family of periodic trajectories. Moreover, there is a $c_{*}>1.4\left(c_{*} \approx 1.47\right)$ such that for $c \in\left(1.4, c_{*}\right)$ the period function for $X$ is not monotone.

2. Proof of Theorem A. For $1<c<2$ the phase portrait of the quadratic system $X$ has the following features. There is a center at the origin. This follows at once from the linearization at the origin and the symmetry of the vector field with respect to the $x$-axis. There is a hyperbolic saddle at $(1 / c, 0)$, a hyperbolic source at $(1, \sqrt{(c-1) / 2)}$ and a hyperbolic sink at $(1,-\sqrt{(c-1) / 2)}$. The line $x=1$ is invariant and most importantly for us the hyperbola $H$,

$$
6 y^{2}=6 c x^{2}-4(c+1) x+(c+1),
$$

is invariant. This integral was found using the general integrals provided by Lunkevich and Sibirskii [7]. We note that $H$ passes through the source and the sink, while its left-hand branch forms the outer boundary of the family of periodic trajectories surrounding the origin. To see this we consider the extension of our vector field $X$ to the Poincaré sphere (the change of coordinates $u=y / x, z=1 / x$ ) obtaining, after multiplication by $z$, in the left half plane chart the system

$$
\dot{u}=z+u^{2} z+u^{2}-c, \quad \dot{z}=-u z+u z^{2} .
$$

In these coordinates it follows easily that there are hyperbolic saddles at $( \pm \sqrt{c}, 0)$ and that our hyperbola $H$ is a separatrix for both saddles which forms the boundary in the finite plane of a separatrix cycle formed from the separatrices of the two saddles with the other portion of the boundary being the trajectory on the line at infinity given by the solution of $\dot{u}=u^{2}-c, \dot{z}=0$ starting at say $(0,0)$. Since the origin is the only zero of $X$ to the left of $H$ in the finite plane it follows that $H$ forms the outer boundary of the family of periodic trajectories surrounding the origin.

We choose $\Sigma$ to be the portion of the $x$-axis given by the interval $(0, L)$ where

$$
L=\frac{2(c+1)-\sqrt{2} \sqrt{(c+1)(2-c)}}{6 c}
$$

corresponds to the left-hand intersection of $H$ with the $x$-axis. We will study the period function $P:(0, L) \rightarrow \mathbf{R}$. Note first that $\lim P(\alpha)=2 \pi$ as $\alpha \rightarrow 0$. Our strategy for showing $P$ is not monotone for $c \in\left(1.4, c_{*}\right)$ consists of two parts. We show $P$ is increasing in some interval $(0, \delta)$ and that $\lim P(\alpha)<2 \pi$ as $\alpha \rightarrow L$.

To see $P$ is increasing for $c>1.4$ near zero we can appeal to the formula for the expansion of $P$ at zero given by Loud [6]. The same formula can also be obtained in the following way. Since $P$ is analytic, $P$ has the form

$$
P(\alpha)=2 \pi+p_{1} \alpha+p_{2} \alpha^{2}+O\left(\alpha^{3}\right) .
$$

After a change to polar coordinates the system becomes

$$
\begin{aligned}
& \dot{r}=r^{2} \sin \theta\left[(1-c) \cos ^{2} \theta+2 \sin ^{2} \theta\right], \\
& \dot{\theta}=1+r \cos \theta\left[\sin ^{2} \theta-c \cos ^{2} \theta\right] .
\end{aligned}
$$

The period function can then be expressed as

$$
P(\alpha)=\int_{0}^{2 \pi} \frac{d \theta}{1+r(\theta, \alpha) \cos \theta\left[\sin ^{2} \theta-c \cos ^{2} \theta\right]},
$$


where $r(\theta, \alpha)$ is the solution of the initial value problem

$$
\frac{d r}{d \theta}=\frac{r^{2} \sin \theta\left[(1-c) \cos ^{2} \theta+2 \sin ^{2} \theta\right]}{1+r \cos \theta\left[\sin ^{2} \theta-c \cos ^{2} \theta\right]}, \quad r(0, \alpha)=\alpha .
$$

Now express $r(\theta, \alpha)$ as

$$
r(\theta, \alpha)=a(\theta) \alpha+b(\theta) \alpha^{2}+O\left(\alpha^{3}\right) .
$$

By substitution into the differential equation for $r(\theta, \alpha)$ we find

$$
\frac{d a}{d \theta}=0, \quad \frac{d b}{d \theta}=a^{2}(\theta)\left[(1-c) \cos ^{2} \theta \sin \theta+2 \sin ^{3} \theta\right] .
$$

Using the initial condition $r(0, \alpha)=\alpha$ we have $a(0)=1, b(0)=0$. A computation then shows

$$
r(\theta, \alpha)=\alpha+\left[\frac{1}{3}(c+1) \cos ^{3} \theta-2 \cos \theta+\frac{1}{3}(5-c)\right] \alpha^{2}+O\left(\alpha^{3}\right) .
$$

Substitution of this expression into the integral formula for $P$ and a computation gives

$$
P(\alpha)=2 \pi+\frac{\pi}{12}\left(10 c^{2}-19 c+7\right) \alpha^{2}+O\left(\alpha^{3}\right) .
$$

The coefficient of $\alpha^{2}$ vanishes at $c=1.4$ and is positive for $c>1.4$. Thus, $P$ increases on some neighborhood of zero.

To complete the proof we show $\lim P(\alpha)$ as $\alpha \rightarrow L$ is less than $2 \pi$. For this we note first that $\lim P(\alpha)$ as $\alpha \rightarrow L$ is precisely the time required to traverse the left-hand branch of the hyperbola $H$. There are several ways to see this fact. One way is to consider the ray $\left\{(r, \theta) \mid r \geq 0, \theta=\theta_{*}\right\}$ in the second quadrant with initial point at the origin which is parallel to the asymptote of $H$ with negative slope (actually, $\tan \theta_{*}=-\sqrt{c}$ ). We have

$$
\begin{aligned}
P(\alpha)= & 2 \int_{0}^{\theta_{*}} \frac{d \theta}{1+r(\theta, \alpha) \cos \theta\left[\sin ^{2} \theta-c \cos ^{2} \theta\right]} \\
& +2 \int_{\theta_{*}}^{\pi} \frac{d \theta}{1+r(\theta, \alpha) \cos \theta\left[\sin ^{2} \theta-c \cos ^{2} \theta\right]}
\end{aligned}
$$

For $\theta_{*} \leq \theta \leq \pi, x=r \cos \theta$ is strictly negative and decreasing. Also, $\left(\sin ^{2} \theta-\right.$ $c \cos ^{2} \theta$ ) vanishes at $\theta_{*}$ and decreases for $\theta_{*}<\theta \leq \pi$. Hence, the integrand of the second integral is positive and bounded by 1 . The limit of this integrand as $\alpha \rightarrow L$ is the function which has value 1 at $\theta_{*}$ and value 0 on $\left(\theta_{*}, \pi\right]$. So, by the Dominated Convergence Theorem, the second integral tends to zero as $\alpha \rightarrow L$. (This fact can also be proven using a $C^{1}$ linearization around the singularity $(-\sqrt{c}, 0)$ at infinity.) Moreover, one can show by a similar application of the Dominated Convergence Theorem that the limit as $\alpha \rightarrow L$ of the first integral is

$$
2 \int_{0}^{\theta_{*}} \frac{d \theta}{1+r(\theta, L) \cos \theta\left[\sin ^{2} \theta-c \cos ^{2} \theta\right]}
$$

which is precisely the time required to traverse the left branch of $H$.

Finally, we show the time required to traverse the left-hand branch of $H$ is less than $2 \pi$ when $c \in\left(1.4, c_{*}\right)$ for some $c_{*}>1.4$. For this we compute the time $T$ using 
the integral $H$ and $d x / d t=(x-1) y$. We have

$$
\begin{aligned}
T & =2 \int_{L}^{-\infty} \frac{d x}{(x-1) y} \\
& =\frac{2}{\sqrt{c}} \int_{-\infty}^{L} \frac{d x}{(1-x) \sqrt{(x-(c+1) / 3 c)^{2}+\left(c^{2}-c-2\right) / 18 c^{2}}} .
\end{aligned}
$$

This integral is elementary. Using the obvious trigonometric substitution we obtain

$$
T=\frac{6 \sqrt{2} \sqrt{c}}{\sqrt{B^{2}-A^{2}}} \ln \left|\frac{\sqrt{B+A}+\sqrt{B-A}}{\sqrt{B+A}-\sqrt{B-A}}\right|
$$

with $A=\sqrt{2+c-c^{2}}$ and $B=\sqrt{2}(2 c-1)$. Since $T(1.4)<2 \pi$ (actually $T(1.4) \approx$ $6.1909)$ there exists a $c_{*}>1.4$ such that $T(c)<2 \pi$ for $c \in\left[1.4, c_{*}\right]$. This completes the proof of Theorem $\mathrm{A}$. In addition, we note that numerical evidence suggests 1.47 is a valid choice for $c_{*}$.

REMARK. For the last part of the proof we could avoid all the computation and rely on another result of Loud in [6] where he determines all quadratic centers where the family of periodic trajectories surrounding the center is isochronous. We could simply show $P^{\prime \prime}(0)=0$ when $c=1.4$ as we have done above. Using Loud's theorem one can check that our system $X$ with $c=1.4$ is not an isochrone. Thus there are three possibilities: $P(L)<2 \pi, P(L)=2 \pi, P(L)>2 \pi$. If $P(L)<2 \pi$ just take $c$, as we did, slightly larger than $2 \pi$ so $P$ increases near zero. If $P(L)=2 \pi$ the periods are not monotone because $X$ at $c=1.4$ is not an isochrone. If $P(L)>2 \pi$ take $c$ slightly less than 1.4. Thus in all cases $P$ is not monotonic.

\section{REFERENCES}

1. C. Chicone, The monotonicity of the period function for planar Hamiltonian vector fields, preprint, 1986.

2. __ Geometric methods for two point nonlinear boundary value problems, preprint, 1986.

3. S. N. Chow and J. A. Sanders, On the number of critical points of the period, J. Differential Equations 64 (1986), 51-66.

4. S. N. Chow and D. Wang, On the monotonicity of the period function of some second order equations, Casopis Pěst. Math. 111 (1986), 14-25.

5. Roberto Conti, About centers of quadratic planar systems, preprint, Instituto Matematico, Universita Degli Studi di Firenze, 1986.

6. W. S. Loud, Behavior of the period of solutions of certain plane autonomous systems near centers, Contributions to Differential Equations 3 (1964), 21-36.

7. V. Lunkevich and K. Sibirskii, Integrals of a general quadratic differential system in cases of a center, Differential Equations 18 (1982), 563-568.

8. F. Rothe, Thermodynamics, real and complex periods of the Volterra model, J. Appl. Math. Phys. 36 (1985), 395-421.

9. __ Periods of oscillation, nondegeneracy and specific heat of Hamiltonian systems in the plane, Proc. Internat. Conf. Differential Equations and Math. Phys. (Birmingham, Alabama, 1986) (to appear).

10. R. Schaaf, $A$ class of Hamiltonian systems with increasing periods, J. Reine Angew. Math. 363 (1985), 96-109.

11. _ Global behaviour of solution branches for some Neumann problems depending on one or several parameters, J. Reine Angew. Math. 346 (1984), 1-31. 
12. D. Wang, On the existence of $2 \pi$-periodic solutions of differential equation $x^{\prime \prime}+g(x)=p(t)$, Chinese Ann. Math. 5A (1984), 61-72.

13. J. Waldvogel, The period in the Lotka-Volterra predator-prey model, SIAM J. Numer. Anal. 20 (1983).

14. $\ldots$, The period in the Lotka-Volterra system is monotonic, J. Math. Anal. Appl. 114 (1986), 178-184.

Department of Mathematics, University of Missouri-Columbia, Columbia, MISSOURI 65211

LimbURgs UNIVERSITAIR CENTRUM, UNIVERSITAIRE CAMPUS, B-3160, DiePENBEeK, BELGIUM 